Zikmund, A.; Ripka, P.

\title{
Calibration of the 3-D Coil System's Orthogonality
}

\author{
A. Zikmund ${ }^{1}$ and P. Ripka ${ }^{1}$ \\ ${ }^{1}$ Faculty of Electrical Engineering, Czech Technical University in Prague, Prague, 16627, Czech Republic
}

\begin{abstract}
Two methods were tested for calibration procedures of the 3-D coil system which is used as a source part of the magnetic tracker. Precise magnetometer's positions with respect to the coil system were used for determining of the nonorthogonality of the coil system as well as for determining of the magnetic moment. The needed number of the reference positions was measured to calculate six unknown calibrated parameters. In comparison, the 3-D coils system was also calibrated with a method which employs the orthogonal homogenous ac field generated in Helmholtz coils. The misalignment of up to two degrees was discovered.
\end{abstract}

Index Terms-Angles, calibration, coils, distance meter.

\section{INTRODUCTION}

$\mathbf{T}$ HE MAGNETIC distance meter contains coils as sources of the magnetic field which is sensed by a three axial magnetic sensor. The distance is calculated with nonlinear optimization from measured magnetic values. Since the magnetic dipole shape of the generated magnetic field is supposed, the coils have to be organized in that orientation to cover the second Gauss position. This can be achievable by a perpendicular arrangement of three coils and thus the second Gauss position of one coil is eliminated by the first Gauss position of another coil. This setup is difficult to manufacture with high precision as the positions of the individual turns in multiturn coil are not precisely known.

The arrangement was also presented in [1] where we observed the different calculated results during a rotation test procedure. During this test the distance between the source coil and the sensing head was kept constant, the coil system was rotated along the axis of one coil at the interval of 0 to 360 degrees and the distance was calculated in each 22.5 degrees. A plotted graph which is depicted in the Fig. 1 (the distance depending on an angle of rotation) discovered a few errors which affected the established distance. In ideal case, we expect this dependence to be constant.

Even when the corrections for magnetic moment and noncoincident coil centers were included into the calculation, the calculated distance was still affected by a certain kind of an imperfection which caused a sinus curve in the graph obtained by rotation procedure.

The only imperfections which were not taken into account and which could be applied were nonorthogonality of the source. We decided to reveal the angular error of source coils by calibration.

\section{Calibration Based on the Reference Positions}

\section{A. Principle}

As it has been already mentioned, the magnetic distance sensor calculated the position according to the measured

[

Color versions of one or more of the figures in this paper are available online at http://ieeexplore.ieee.org.

Digital Object Identifier 10.1109/TMAG.2012.2218583

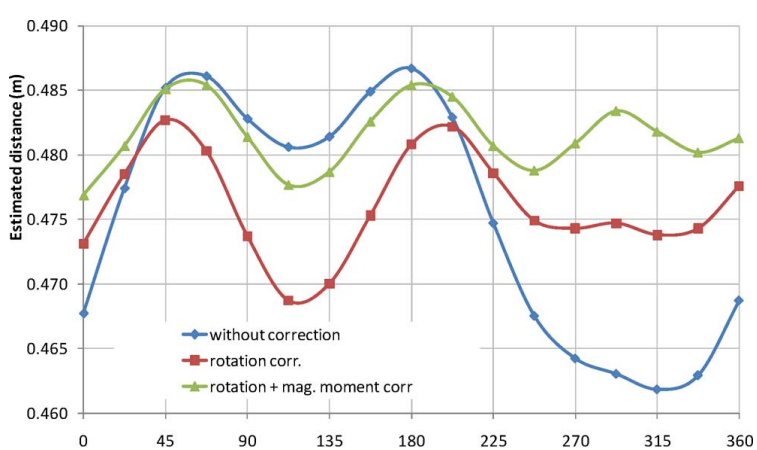

Fig. 1. Rotation procedure which reveals the system imperfestidep otation (deg)

magnetic field. This calibration is the opposite method to the magnetic distance sensor principle.

Reference points at very precisely defined positions are selected. The reference points are spread into near area of the coil system to reach strong magnetic signal measured with the magnetometer and to suppress the noise. In each position labeled with number 1 to 5 in Fig. 2, the magnetometer has to be oriented the same way to avoid the necessity of rotating the angular correction. The coils are successively excited and the magnetic field measured with the magnetic sensor is recorded in each reference positions.

After completing all reference points, the set of the measured magnetic fields is collected and sent to the optimization algorithm which establishes the coil system's parameters.

Since we have a sufficient number of the values that are measured in the reference positions, we are also able to calculate the magnetic moments of the individual coils.

Therefore, seven unknown parameters are supposed to be established. Three of them are the magnetic moments of the coils and the other four are two rotation angles for each of the two coils (if the alignment of third coil is declared).

The positions of the reference points have to be measured with millimeter precision.

The manufactured coil system (see Fig. 4) does not have identical centers of the coils. This fact has to be included into the correction algorithm because the position offsets affect the calculated parameters.

\section{B. Magnetic Sensor}

The important part of this calibration method is a tri-axial magnetic sensor that measures the complete vector information 


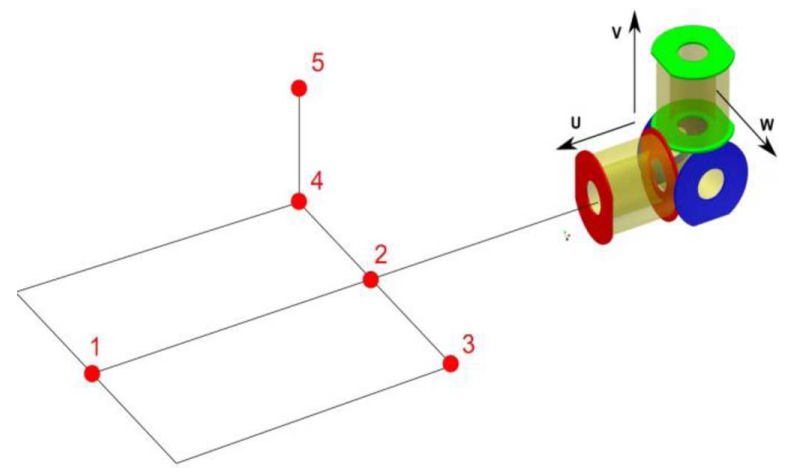

Fig. 2. Sketch of the calibration method based on five reference positions labeled with numbers 1 to 5 .
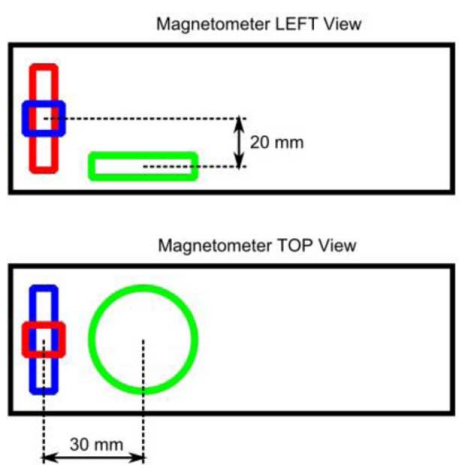

Fig. 3. Noncoincided centers of magnetometer's cores (blue Y axis, red Z axis and green $\mathrm{X}$ axis).

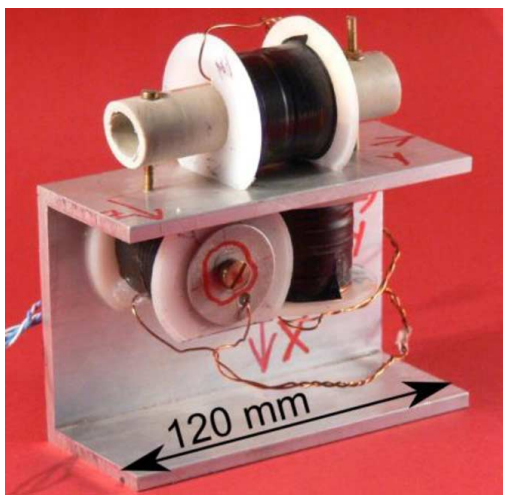

Fig. 4. Calibrated 3-D coil system.

of the generated magnetic field. To get precise calibrated values, the magnetic sensor should have low noise. Offset variations is suppressed by using bipolar excitation field.

Magnetoresistive sensors are small and cheap, but they have a noise level in order of unit $\mathrm{nT}$ [2] which is too high. Therefore we decided to use axial fluxgate sensors (Billingsley TFM100G2) with resolution of $0.1 \mathrm{nT}$ and sufficient offset and gain stability.

During testing of the calibration procedure we found that other corrections should be introduced. The used tri-axial fluxgate magnetometer sensor contains 2 ring cores. Two axes ( $Z$ and $\mathrm{Y}$ ) are measured using the first core. The third axis is measured by a separate core which means that the third axis has not the same center as the $\mathrm{Z}$ and $\mathrm{Y}$ axes. according to picture in the Fig. 3, the position offset of the $\mathrm{X}$ axis was in our case was corrected with the value of 30 or $20 \mathrm{~mm}$ respective.
TABLE I

Calculate Misaligned Angles From the Positioning Procedure

\begin{tabular}{cc}
\hline \hline Coils & Angle \\
& \\
\hline $\mathrm{U}-\mathrm{V}$ & 91.4 \\
$\mathrm{U}-\mathrm{W}$ & 85 \\
$\mathrm{~V}-\mathrm{W}$ & 87.5 \\
\hline \hline
\end{tabular}

An excitation sequence is similar to the one published in [1]. Current square wave pulses of both polarities are sequentially sent to individual source coils $\mathrm{U}, \mathrm{V}$ and $\mathrm{W}$. In this way, we can easily remove the Earth's magnetic field vector and, by averaging from several cycles, we can also reduce the noise. To keep the eddy current effect low, the operating frequency is below 10 $\mathrm{Hz}$.

\section{Calculation}

The calibrated parameters of the coil system are calculated by the algorithm that is based on the Levenberg-Marquardt nonlinear optimization method [3] because spatial rotations of the magnetic coil are estimated from the nonlinear equations which cannot be solved analytical way.

\section{Results}

Table I presents the calculated angular misalignment of the coil system shown in the Fig. 4. The angles are recalculated from yaw, pitch and roll to the notation comparable with further method and thus the misaligned angles are transformed to the plane defined by two neighbor coils.

\section{Calibration Based on the Homogenous aC Field}

\section{A. Principle}

To verify the calculated results from the previous method, we decided to calibrate the misaligned angles also in homogenous ac magnetic field.

The method uses the homogenous field generated in the Helmholtz coils. The assumption of the homogenous magnetic field in space where the coil system is calibrated brings simplifications in comparison to the previous method.

First of all, the position offsets of the individual coil can be neglected because the magnetic field has the same orientation and size in the certain space of a Helmholtz coil.

Additionally, if a Helmholtz coil is three dimensional with calibrated orthogonality [4], the calibration is more simple because the misaligned angle can be investigated using the perpendicular coil without using the rotation. The differences of the sensitivities of the calibrated coils are omitted since the angle is calculated only from the coil independently.

The Helmholtz coil is excited by the ac current with frequency of $12 \mathrm{~Hz}$ to avoid the eddy current and the coils of the calibrated coil system serve as a pick-up coil.

The voltage induced in the coil is proportional to the sine function of the angle. The method is depicted in the Fig. 5.

To get a precise measurement, we use a lock-in amplifier that measures the induced voltage at the calibrated coils and is 


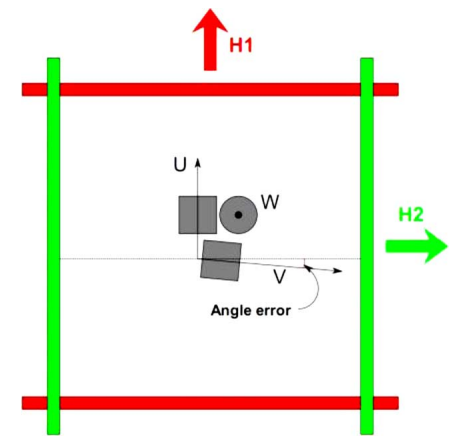

Fig. 5. Sketch of the calibration method based orthogonal Helmholtz coil.

TABLE II

Calculate Misaligned Angles From the Homogenous Field PROCEDURE

\begin{tabular}{cc}
\hline \hline Coils & Angle \\
\hline U-V & 91.2 \\
U-W & 89.1 \\
V-W & 88.5 \\
\hline \hline
\end{tabular}

TABLE III

Calculate Misaligned Angles From the Homogenous Field Procedure With an ARTIFICIAL ERROR

\begin{tabular}{cc}
\hline \hline Coils & Angle \\
\hline U-V & 93.8 \\
U-W & 89.1 \\
V-W & 88.5 \\
\hline \hline
\end{tabular}

locked at the exciting frequency. In this way we are able to measure very weak signals because the induced voltage is around unit of micro volts.

\section{B. Procedure}

One of the Helmholtz (labeled as $\mathrm{H} 2$ in the picture) coils is excited by the ac current and the calibrated coil system is put into the middle of its homogeneous space. One coil ( $\mathrm{U}$ in the picture) of calibrated system is chosen as a reference one and the minimum of its induced voltage is found by rotating of the calibrated coil system. To search the minimum is much more precise than to search the maximum because there is the sine curve flat. If the minimum value is reached that means the reference coil is perfectly 90 degrees aligned to the excited Helmholtz coil.

Then the perpendicular Helmholtz coil (H1) is excite which generates the magnetic field rotated by 90 degrees according to calibrated system. The minimum of the induced voltage should be on the orthogonal coil (V) but due to angular imperfection it is not zero and based on the induced voltage, the angular misalignment can be calculated according to an equation

$$
\alpha=\operatorname{arctg}\left(\frac{U_{0 \mathrm{deg}}}{U_{90 \mathrm{deg}}}\right)
$$

where $\mathrm{U}_{0 \text { deg }}$ is a induced voltage from the perpendicular coil and $\mathrm{U}_{90 \mathrm{deg}}$ is a induced voltage if the aligned coil is excited.

\section{Results}

The angular misalignments measured by this method are shown in the Table II.

\section{Artificial Angular Error}

In order to test the whole procedure we introduced error by rotating the $U$ coil by 2 degrees. Then we carried out the same calibration procedure and we calculated new updated values which are shown in the Table III.

The corresponding angle which was added between $\mathrm{U}$ and $\mathrm{V}$ coil was discovered by this procedure. The angle was changed from 91.2 degrees to 93.5 which gave us the exact additional error.

\section{CONCLUSION}

The first method which uses the precisely defined positions is applicable in situations when the Helmholtz coils are not available.

The Helmholtz coil procedure is definitely more accurate in terms of calculating angular misalignment because:

- the coil constant can be neglected because the induced voltage depends only on the angular orientation

- the position offset of the coil is neglected due to the homogenous field of the Helmholtz coils

On the other hand, the first method estimates all parameters which should be corrected during normal function of the distance sensor. For example, the offset of the magnetometer cores should be also taken into account during the calculation carried out in the distance meter.

We have to add that the first method is less accurate because it is difficult to determine all parameters mentioned above. If we simulated the influence of these parameters we got the huge dependency on the calculated results. If we change e.g., parameter of the fluxgate core offset position by $5 \mathrm{~mm}$, the effect on calculated angles is 0.5 degrees.

This first method could be improved if we use the different kind of magnetometer to avoid the mentioned problem with core offsets.

\section{ACKNOWLEDGMENT}

This work was supported by Research Grant GA102/09/ H082 sponsored by Czech Science Foundation and Grant SGS12/194/OHK3/3T/13 sponsored by Czech Technical University in Prague.

\section{REFERENCES}

[1] A. Zikmund and P. Ripka, "A magnetic distance sensor with high precision," Sens. Actuators A, Phys, vol. 186, pp. 137-142, May 2012.

[2] P. Ripka, M. Vopalensky, A. Platil, M. Doscher, K.-M. H. Lenssen, and H. Hauser, "AMR magnetometer," J. Magn. Magn. Mater., no. 254-255, pp. 639-641, 2003.

[3] D. Marquardt, "An algorithm for least-squares estimation of nonlinear parameters," SIAM J. Appl. Math., vol. 11, pp. 431-441, 1963.

[4] A. Zikmund and P. Ripka, "Scalar calibration of the 3-D coil system," J. Elect. Eng., vol. 61, pp. 39-41, 2010. 